\title{
Torri, corsari e contrabbandieri in Calabria Ultra durante il Decennio Francese (1806-1815)
}

Towers, corsairs and smugglers in Calabria Ultra during the French Decade (1806-1815)

\section{Vincenzo Cataldo}

Università degli Studi di Messina, Messina, Italy, vincataldo@tiscali.it

\begin{abstract}
At the beginning of the nineteenth century, even if the phenomenon of running war had subsided, the watch towers still had an active role in controlling the coasts of Southern Italy. Under the French administration some of them were assigned to customs posts, others continued to report the corsair boats always ready to carry out incursive actions. Merchant ships, fishermen and peasants were still struck by the devastating Turkish-Barbarian cruises, but also by corsairs armed by the British in an eternal struggle against the French. The towers are regularly guarded by sentinels armed with non-military weapons, which are not functional to the increasingly sophisticated assaults of the Corsair marines. The people in charge of the customs had to manage a staff often absent from the guardhouse due to malarial fevers, especially during the summer when the coasts were excessively hot. The customs documentation shows the economy of a Southern Italy still rooted in the classic export products: oil, dried figs, cotton, cheese, wine and coarse wool cloths. Raw silk is absent from the market, one of the most exported products until the second half of the eighteenth century and supplanted by the olive tree.
\end{abstract}

Keywords: Mediterranean, towers, corsairs, smuggling, trading.

Agli esordi dell'Ottocento la guerra di corsa e l'atavico fenomeno del contrabbando, anche se diminuiti, incidevano ancora negativamente nel tessuto commerciale del Regno di Napoli ${ }^{1}$. La pirateria barbaresca si era attenuata per lasciare posto agli altrettanti assetati equipaggi occidentali (francesi, inglesi, maltesi e siciliani), sempre pronti ad intercettare lungo le coste del Mediterraneo centrale navigli carichi di mercanzie destinate soprattutto alla capitale. Il governo non disponeva di una flotta capace di controllare e intercettare in maniera sistematica e, soprattutto, efficace le navi corsaro-piratesche. Risultava, quindi, facile per gli equpaggi corsari attendere dietro un promontorio o un'insenatura la preda di turno che in pochi minuti sarebbe stata sottoposta ad uno sfrenato abbordaggio. I comandanti corsari occidentali, a differenza di quelli turcobarbareschi, non manifestavano nessun interesse alla cattura e alla, conseguente, schiavizzazione degli equipaggi; quanto invece all'incettazione delle merci molto più facilmente piazzabili nei mercati paralleli.

Spazzate via o, comunque, diminuite le crociere turco-barbaresche, il Mediterraneo diventò lo spazio dove si affrontarono, in una continua guerriglia sottile, le armate francesi e inglesi supportate dai loro alleati. La guerra di corsa e la pratica del contrabbando divennero in questo modo una contrapposizione di natura economica, politica e militare nelle dinamiche di un conflitto di più vasta portata, inteso come strumento per il controllo di aree vitali in funzione della propria potenza. 
Durante il Decennio francese (1806-1815), i francesi misero in campo delle forze attive costituite da reparti specializzati chiamati brigate, utilizzati per la custodia dei litorali (Caldora, 1960; Mozzillo, 1972). Le brigate espletavano servizio per conto delle dogane. In Calabria Ultra la Direzione delle Dogane aveva sede a Catanzaro. Reggio era sede di Ispezione; a Gerace vi era l'ufficio di Controleria competente da Capo Spartivento a Capo Stalettì.

La dogana era l'amministrazione incaricata di percepire i tributi saldati sia all'entrata che all'uscita delle merci. Con la medesima definizione si intendevano i dazi pagati e i locali dove l'amministrazione aveva sede. La guardia era formata dai controlori di brigata, tenenti d'ordine, brigadieri e preposti a piedi o a cavallo, piloti, sottopiloti e marinai.

La documentazione dell'ufficio doganale offre una serie di spunti per capire le difficoltà incontrate dal governo franco-napoletano nella gestione dei mari di propria competenza ${ }^{2}$.

La legge stabiliva che i diritti di immissione, estrazione o circolazione delle merci potevano essere percepiti solo nei burò prestabiliti alle frontiere di terra e di mare ed in quelle all'interno del Regno. Le frontiere erano finalizzate a servire da seconda linea, come punti di controllo delle merci e per riscuotere i diritti determinati dalla tariffa particolare sulle mercanzie.

L'assunzione da parte dei vari porti, fondaci e spiagge di regolamenti doganali propri, comportò una confusione legislativo-fiscale con conseguenze negative sul commercio, tali da far proliferare il contrabbando. La maggior parte del traffico illegale veniva compiuto da benestanti, patroni di barca e sacerdoti, come dimostra l'operazione di polizia effettuata in alcuni casali di Reggio dove nel giugno 1808 furono sequestrati circa 700 carichi di olio, anche se poi fu dimostrato dal burolista di Villa che solo una parte della merce risultava contrabbandata poiché molte partite di olio confiscato erano provviste di polizze e, pertanto, dovevano essere liberate $^{3 .}$ A riguardo, i negozianti napoletani Gio- vanni Maria Paturzo e Gennaro Fiodo si ritennero lesi dai loro diritti commerciali perché quell'olio era stato comprato nei paesi della Piana di Gioia e riposti nei magazzini della cintura urbana reggina nell'attesa di essere spediti verso la capitale e l'estero. ${ }^{4}$

A differenza di quanto avveniva nel Settecento, Reggio dimostra una accentuata capacità di intraprendere iniziative commerciali verso l'area adriatica, come dimostra Natale Sciarrone che nel 1808 imbarcò 39 salme di olio dirette ad Ancona e a Venezia ${ }^{5}$. Nel complesso, le navi giungevano e ripartivano dai porticcioli del litorale jonico principalmente verso Taranto, Crotone, Napoli e il Golfo di Venezia con diverse tipologie di merci.

A turbare la pace dei calabresi in età napoleonica furono molti corsari armati dagli inglesi. Nelle loro apparizioni, i predoni britannici utilizzarono una violenza ancora superiore a quella turco barbaresca, essendo i loro equipaggi costituiti da ex galeotti e avventurieri. A Cetraro, il 7 giugno 1806 tre imbarcazioni inglesi inseguirono due barche regnicole cariche di olio. Durante il conflitto perirono molti inglesi e due dalla parte dei volontari cetraresi (Savaglio, Capalbo, 2004, pp. $112,113,114)$. Nelle acque di Siderno un brich inglese e due lance si misero ad inseguire ed a sparare contro quattro barcelle $e^{6}$ napoletane i cui equipaggi, come da prassi, abbandonarono gli scafi. Il fuoco delle guardie disposte lungo il litorale riuscì a fugare $\mathrm{i}$ corsari ${ }^{7}$.

Le torri di guardia, che fino alla fine del Settecento avevano rappresentato una formidabile rete anticursiva, si erano trasformate in parte in posti doganali da dove espletare non solo il controllo litoraneo, ma anche le operazioni commerciali. Nella fascia jonica reggina, le torri attive erano quelle di Stalettì, Soverato, S. Andrea, Badolato, S. Caterina, Monasterace, Riace, Castelvetere, Roccella, Gioiosa, Siderno, Gerace, Bovalino, Bianco, Bruzzano. Dai rapporti compilati durante il servizio di ispezione delle Dogane Reali, il controloro di Gerace Matteo Princi ritrovò le torri da Brancaleone a Soverato regolarmente vigilate, anche se le sentinelle erano dotate di armi obsolete. 


\begin{tabular}{|l|l|l|l|l|l|}
\hline $\begin{array}{l}\text { Posto di Con- } \\
\text { troleria }\end{array}$ & Abitazioni & Proprietà delle abitazioni & $\begin{array}{l}\text { Fitto in } \\
\text { ducati }\end{array}$ & $\begin{array}{l}\text { Rata del } \\
\mathbf{f i t t o}^{\mathbf{8}}\end{array}$ & Imposta9 $^{\mathbf{9}}$ \\
\hline Brancaleone & Piano superiore & Duca di Bruzzano & 3 & & \\
\hline Bianco & Casetta terrana & Regia Corte & & & \\
\hline Bovalino & Casetta terrana & Vedova Sergente Schiariti & 3 & & \\
\hline Gerace & Casetta terrana & Pietro Triunveri & 3 & & \\
\hline Siderno & Piano superiore & Dr. fisico Giuseppe Bello & 4 & & \\
\hline Gioiosa & Piano superiore & Marchese di Arena & 4 & & \\
\hline Roccella & Piano superiore & Regia Corte & & & \\
\hline Castelvetere & Nella regia torre & Regia Corte & & & \\
\hline Riace & Piano superiore & Marchese Gagliardi & 4 & & \\
\hline Monasterace & Piano superiore & Barone Oliva & 4 & & \\
\hline S. Caterina & Casa & Massaro Criniti & 3 & & \\
\hline Badolato & Piano superiore & Sig. Paparo & 4 & & \\
\hline S. Andrea & Piano superiore & Sig. Saverio Calabretti & 3 & & \\
\hline Soverato & Nella regia torre & Regia Corte & & & \\
\hline Stalettì & Nella regia torre & Regia Corte & & & \\
\hline
\end{tabular}

Tab. 1. Stato delle abitazioni dei posti di guardia della Controleria di Gerace anno $1808 .{ }^{10}$

Del mese di febbraio del 1808 la fornitura dell'olio nel comparto compreso tra la torre di Stalettì fino a quella di Capo Bruzzano aveva segnato un esito di 288 carichi. Già dalla seconda metà del Settecento l'olio d'oliva era diventato uno dei prodotti principali d'esportazione, soprattutto in Francia ed Inghilterra per alimentare i saponifici e le industrie ${ }^{11}$. Proprio agli inizi dell'Ottocento il comparto olivicolo risultò l'industria più ricca della Calabria, scardinando il primato detenuto fin a quel momento dal settore serico (Bevilacqua, 1987, p. 25; Jannucci, 1981). La richiesta di olio, sostenuta dal mercato internazionale, stimolò i corsari e i contrabbandieri a ricercare questo prodotto. Tra l'altro, il mercato meridionale relativo a questo periodo viveva una fase di flessione nell'esportazione a causa dell'interruzione del traffico commerciale dovuto ai crescenti conflitti tra le potenze europee.

Nelle pieghe delle crisi si insidiavano contrabbandieri e gente di varia risma. Nel 1808 alcuni briganti di Ferruzzano, da più tempo rifugiati in Sicilia, nottetempo erano ricomparsi decisi ad intercettare carichi di olio. Dopo un conflitto a fuoco con i guardiacoste, i banditi si rifugiarono nei boschi di Rudina e Dedera.

L'intervento anche della guardia civica di Bianco e di Ardore portò ad un ennesimo scontro a fuoco per ostacolare il loro tentativo di reimbarcarsi.

La superficie liquida calabrese era battuta costantemente da equipaggi corsari francesi con l'obiettivo soprattutto di interdire il rifornimento di olio alle industrie inglesi. Il 6 luglio 1808 nei pressi di Capo Bruzzano si presentò una squadra di navigli corsari francesi. Una di esse fece fuoco su una palandra ${ }^{I 2}$, tra l'altro già predata, e a nulla valse l'intervento della fucileria della brigata guardacoste e dei volontari di Bianco, poiché la nave pirata si era portata già fuori tiro. ${ }^{13}$

Dai caricatoi della Riviera jonica si spedivano olio, fichi secchi, cotone, formaggi, vino ed $a r$ - 
baso $^{14}$. Ogni tanto si intravedeva il passaggio di convogli di grano provenienti da Crotone diretti verso Napoli e non era raro che, spinti dal timore di essere assaltati da feluche nemiche, si rifugiassero nei pressi delle torri, come successe ad una barcella approdata alla marina di Bovalino, intimorita da una flotta di 32 velieri avvistati a Capo Spartivento ${ }^{15}$. Il pericolo di essere predati era in effetti sempre incombente: la barca di padron Tafuri, carica di olio, diretta verso la capitale, nel 1812 fu intercettata nelle acque tra Rosarno e Capo Vaticano da un veliero corsaro (Liberti, 2000, p. 4).

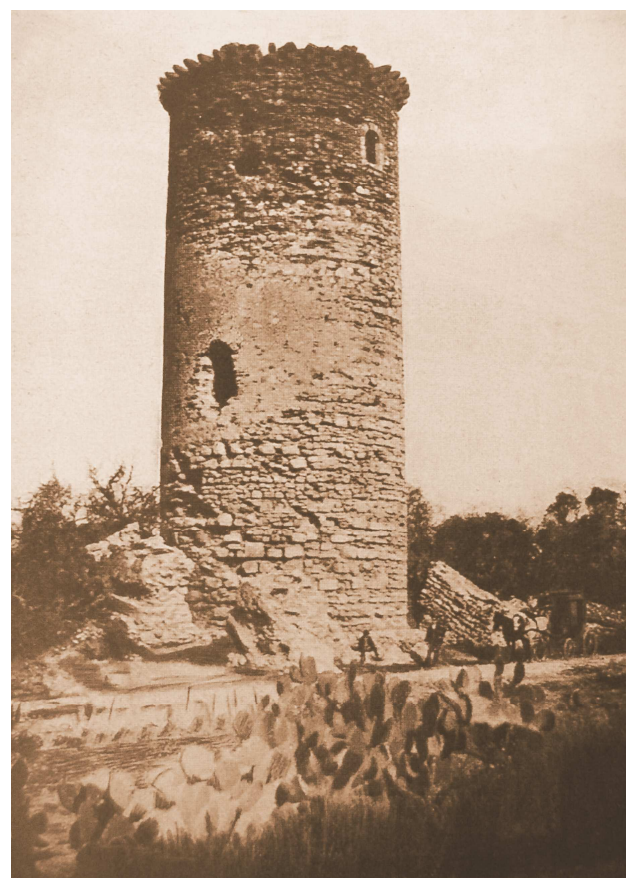

Fig. 1. La torre di Pagliopoli di Gerace Marina prima del terremoto del 1908.

I responsabili delle Dogane dovevano gestire un personale spesso assente dal posto di guardia a causa delle febbri malariche, diffuse specialmente durante il periodo estivo quando i litoranei si presentavano infetti “da un' aria crassa, e pestifera"16 in posti scomodi, disabitati ed eccessivamente caldi. Gli ufficiali trovavano molte difficoltà a rimpiazzare gli agenti di guardia, spesso ammalati o che volontariamente si ritiravano nei loro paesi per paura della terzana.
Il 15 maggio 1808 due imbarcazioni corsare siciliane, dopo aver cannoneggiato la torre di Palizzi, al largo di Bova aggredirono due barcelle cariche di grano e formaggio provenienti da Crotone. Il fuoco intenso direzionato dalle brigate, indusse le navi corsare ad abbandonare il tentativo di arrembaggio. Il successivo 24, nei pressi di Bianco, un brich inglese si pose a dare la caccia a due barcelle provenienti da Reggio e un'altra in arrivo da Crotone carica di grano. Le tre imbarcazioni si rifugiano sotto torre Scinusa di Bovalino, protette dal fuoco di sbarramento della brigata e della guardia civica del centro jonico.

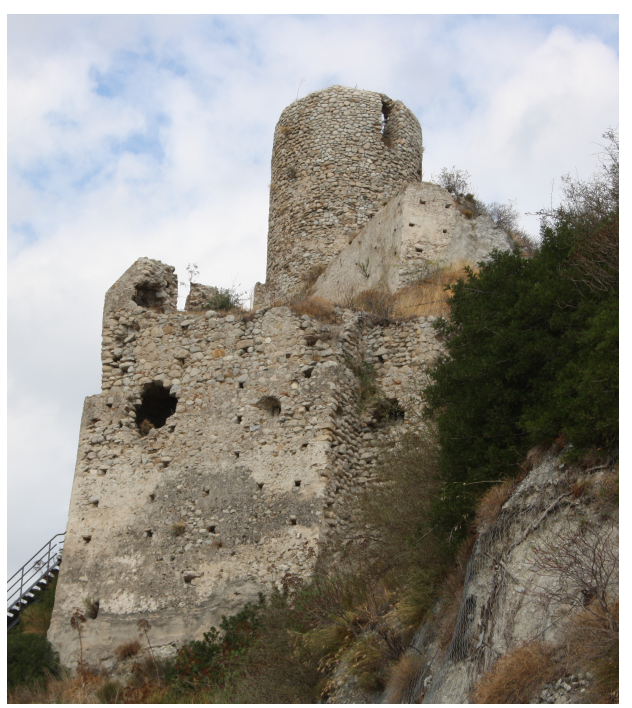

Fig. 2. La torre di Roccella.

Ogni brigata era formata da non più di quattro elementi, dei quali due erano assegnati nei burò per servizio della dogana; per cui rimanevano altri due. Considerato che uno di loro partiva con i plichi, la brigata rimaneva composta da una sola persona. In queste situazioni potevano accadere dei disordini, per cui il controloro Matteo Vinci chiese un aumento dei guardacoste a servizio delle brigate e la dotazione di un valido munizionamento. Ma nel servizio delle brigate era carente anche la comunicazione. Un mancato rapporto aveva impedito di intervenire in maniera adeguata nel mare di Capo Bruzzano ad osteggiare l'attacco di una galeotta e di una speronara corsare a danno di una sciabaca del luogo. ${ }^{17}$ 


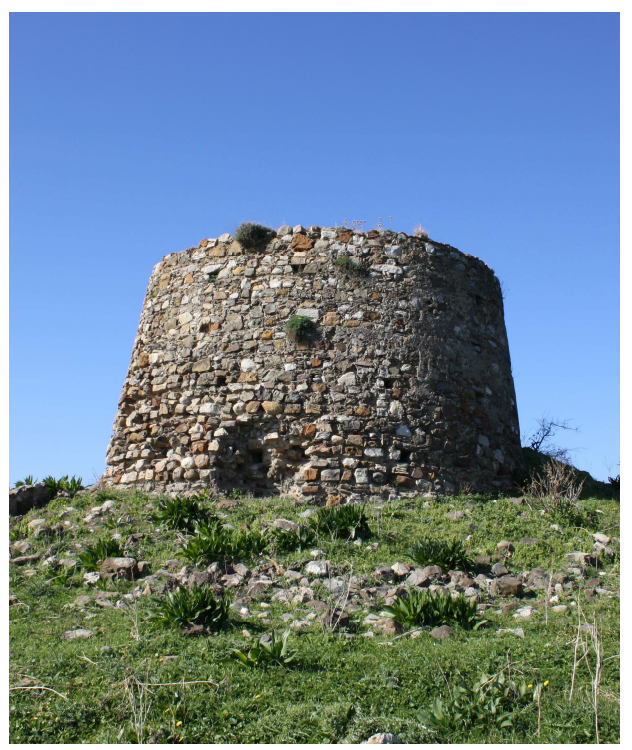

Fig. 3. La torre di Capo Bruzzano.

Nelle traversate piuttosto lunghe, considerati i tempi dilatati di navigazione, si faceva più probabile, specie nei mesi autunnali e invernali, il rischio di impetuose tempeste. Il 17 ottobre 1808 a Bovalino arrivarono sei membri di equipaggio di un trabaccolo posto a due miglia dalla riva. Il ventiduenne maltese Marcantonio Manatti raccontò di essere partito dall'isola 50 giorni prima con un equipaggio di 45 marinai comandato dal capitano corsaro Wallovich. Arrivato nel Golfo di Venezia, nel canale di Zara predarono il trabaccolo comandato da patron Santo Mancini di Pesaro, carico di olio e capperi all'aceto; dopodiché, il capitano incaricò il suo secondo a condurre la nave a Malta con altri cinque marinai provenienti dalla stessa isola, dalla Grecia e dall'Austria. A Capo Spartivento, incontrata una tempesta di mare, il giovane comandante fu costretto a gettare una parte del carico per alleggerire l'imbarcazione. Qualche giorno dopo a Capo Stilo un'altra tempesta ruppe il timone costringendo l'equipaggio ad abbandonarsi alla direzione dei venti; finché non si ritrovarono al largo di Bovalino con lo scafo malconcio.

Intanto lungo le coste dello Jonio meridionale continuava il passaggio di navi mercantili. L'8 agosto 1808 nella spiaggia di Monasterace giunse una barcella per caricare olio destinato alla capitale; a Siderno una barcella arrivò dalla costa napoletana carica di pasta e di altre merci. La stessa avrebbe imbarcato olio da condurre a Napoli. Dallo stesso scaro di Monasterace l'11 agosto furono spediti via mare otto botti di olio e altri generi; via terra capperi non salati, olio e 40 rotola di formaggio. A Villa approdò una nave con un carico di cuoio peloso, zucchero rosso, campece, sarde salate ed altre merci. Nelle spedizioni del burò di Soverato vengono annotate quattro spedizione di pietra-gesso per Taranto, molte estrazioni di olio via terra, una spedizione di tela cotonina, sarde salate via terra ed una immissione di 40 cuoi pelosi e di 70 rotoli di riso da Villa.

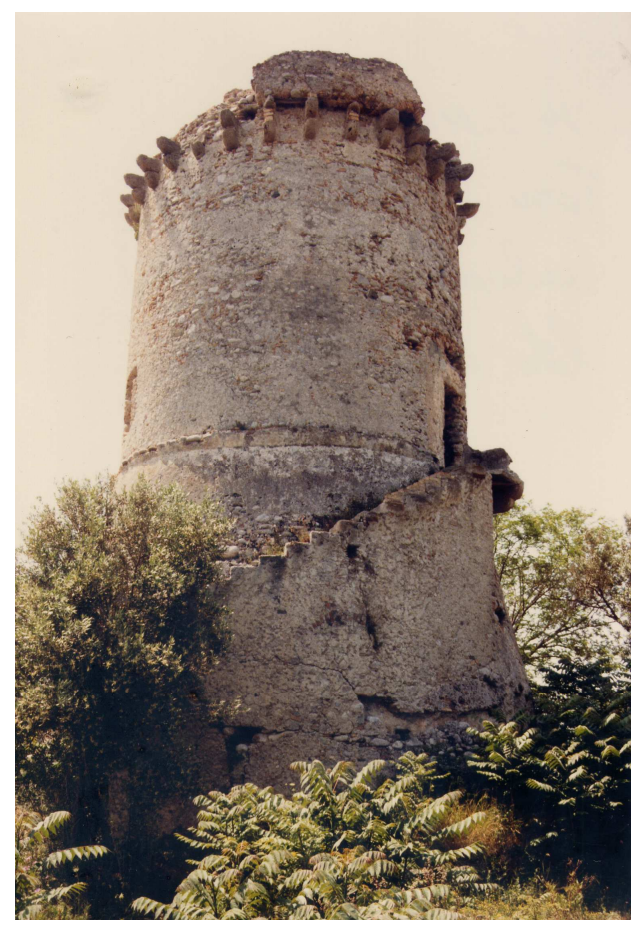

Fig. 4. La torre di Castelvetere (odierna Caulonia).

Tra le pieghe dei rapporti si notano casi di estorsioni compiute dai guardiacoste a danno di commercianti e passeggeri. Il governatore di Badolato Falletti denunciò il sottotenente guardacosta Gagliardi di Santa Caterina di continue estorsioni nei riguardi dei passeggeri, sotto pretesto che portassero merci soggette ai diritti doganali. ${ }^{18}$ 


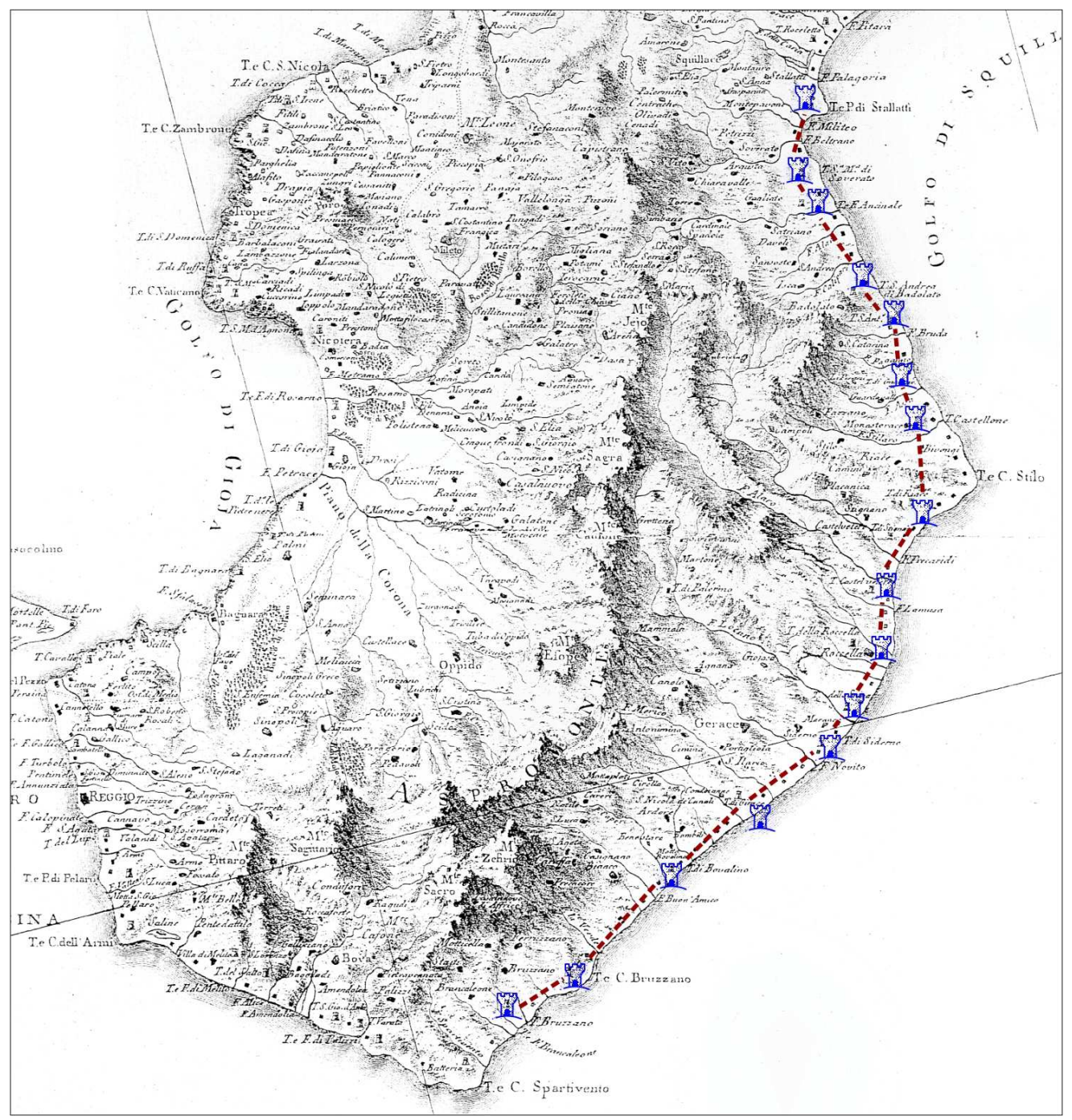

Fig. 5. Nuova Carta Geografica della Calabria Ultra, Ing. Luigi Ruel (1784-1786).

Paradigmatico è anche il rapporto del tenente Aniello Lanzetta circa le angherie compiute dal sottotenente Sacco di Palizzi nei confronti di padron Giovanni Maresca di Positano, giunto a Roccella con la sua barcella dalla costa amalfitana carica di pasta e di porcellana, "genere di buonissima qualità". Il Sacco aveva praticato nei confronti del capitano positanese "le più atroci angariamenti, e che il medesimo si prese alla fine due rotoli di pasta, e da un'altra barcella una dozzina di porcellana" ${ }^{\prime 19}$. Questi disonorevoli atteggiamenti, affermava il Lanzetta, "ledono la reputazione de' buoni, ed il Corpo acquista mal nome. Noi che dobbiamo mantenere l'equilibrio di questo poco commercio, e garantirlo per quando le nostre facoltà si estendono, non è di giusto, che ci sentiamo noi stessi l'incaglio, ed il ladroneccio. In questa maniera, distorciamo le benefiche mire del Governo, e ci rendiamo peggiori de' Briganti”.

Il controloro Princi annotava ancora l'arrivo di una barcella napoletana diretta nella marina di Stilo a caricare olio da destinare alla capitale. A 
Bianco approdarono tre imbarcazioni cariche di grano provenienti da Crotone e dirette a Reggio. Nel posto di Soverato ritrovò due barcelle tarantine giunte per vendere merce; altre due cariche di grano destinato a Reggio; mentre a Siderno giunse una barcella carica di sale proveniente da Crotone. Nel solo mese di maggio 1808 dalla dogana di Gerace furono imbarcate 39000 schiocche di fichi. La parte preponderante giunse da Gerace e Canolo e in misura minore da Casalnuovo. Cifre ancora più modeste pervennero da Grotteria, Soriano, Martone, Serra e Oppido; mentre quasi insignificante appare il commercio di olio (11 cafisi provenienti da Bianco e Bruzzano $)^{20}$. Da Crotone arrivarono due barcelle di grano, da Amalfi altre due imbarcazioni cariche di carta e porcellana, mentre da Villa zucchero e caffè. ${ }^{21}$

Con il ritorno dei Borbone, dal 1816 la guardia delle coste fu affidata principalmente ai controlori delle Dogane Reali. Ogni brigata aveva nel suo seno delle scorridoie di servizio composte da un numero variabile, dipendente dal settore litoraneo da controllare.

\section{Note}

1 Fontenay, Tenenti, 2006. Per la Calabria l'argomento è stato ampiamente trattato in $\mathrm{Ca}$ taldo, 2014. Allo stesso testo si rimanda per l'ampia bibliografia internazionale.

${ }^{2}$ Archivio di Stato di Reggio Calabria (d'ora in poi ASRC), Fondo Dogane.

${ }^{3}$ ASRC, Fondo Dogane, b. 5, fasc. 438.

${ }^{4}$ ASRC, Fondo Dogane, b. 2, fasc. 176, f. 1r.

${ }^{5}$ ASRC, Fondo Dogane, b. 5, fasc. 410, f. 1r, Reggio 3 agosto 1808.

${ }^{6} \mathrm{La}$ barcella è una variante della bilancella ligure utilizzata durante l'Ottocento come nave da pesca, ma anche da trasporto come attestato in questo contributo (Dellabarba, Guerreri, 2003, pp. 50-53).

${ }^{7}$ ASRC, Fondo Dogane, b. 6, fasc. 518, Siderno $1^{\circ}$ novembre 1808 .

${ }^{8}$ I fitti delle abitazioni sono tutti rateizzati.

${ }^{9}$ Le voci sono provviste tutte di imposta per un totale di 22:34:2 ducati.

${ }^{10}$ ASRC, Fondo Dogane, b. 6, fasc. 507, anno 1808, Gerace, 31 agosto 1808. Direttore generale delle Dogane delle due Calabrie era il marchese de Riso di Catanzaro (Catanzaro 24 agosto 1808).

${ }^{11}$ Montaudo, 2005. Per il commercio dell'olio in Calabria, Cataldo, 2012a, 2012b, 2015.

${ }^{12}$ Nave larga e scoperta.

${ }^{13}$ ASRC, Fondo Dogane, b. 6, fasc. 535, 1808.

${ }^{14}$ Panno di lana grossolana.

15 ASRC, Fondo Dogane, b. 6, fasc. 535, 1808. Per la produzione e il commercio del grano crotonese; Cfr. Cataldo, 2017, e in corso di pubblicazione.

${ }^{16}$ ASRC, Fondo Dogane, b. 6, fasc. 518, Bovalino 17 aprile 1808 .

${ }^{17}$ ASRC, Fondo Dogane, b. 6, fasc. 535, Bianco 7 luglio 1708.

${ }^{18}$ ASRC, Fondo Dogane, b. 6, fasc. 508.

${ }^{19}$ ASRC, Fondo Dogane, b. 6, fasc. 537.

20 ASRC, Fondo Dogane, b. 6, fasc. 538. Le schiocche sono fichi secchi infilzati su alcuni stecchetti di legno.

21 Nelle osservazioni della precedente tabella viene precisato che ogni cafiso di olio corrispondeva a rotola 13 , apprezzato a 28 ducati la botte. La botte era formata da 37 cafisi. Nei lasciapassare si esigeva solo la tassa sulla carta bollata.

\section{Bibliography}

Bevilacqua, P. (1987). "Il Mezzogiorno nel mercato internazionale (secoli XVIII-XIX)”, Meridiana, 1.

Caldora, U. (1960). Calabria napoleonica (1806-1815), Fiorentino, Napoli.

Cataldo, V. (2012a). "Arrendamenti nel Mezzogiorno del Settecento: il caso Calabria, Archivio storico per la Calabria e la Lucania”, a. LXXVIII, pp. 130-168. 
Cataldo, V. (2012b). Contratti e rapporti di produzione nella Calabria del XVIII secolo, Edizioni Scientifiche Italiane, Napoli.

Cataldo, V. (2014). La Frontiera di pietra. Torri, uomini e pirati nella Calabria moderna, Edizioni Scientifiche Italiane, Napoli.

Cataldo, V. (2015). “L'olivicoltura calabrese nel Settecento", RogeriusXVIII, 2, pp. 29-38.

Cataldo, V. (2017). Crotone nel Settecento. La città, il grano, il mare, Pancallo, Locri.

Cataldo, V. (in corso di pubblicazione). Crotone. Una città al centro del Mediterraneo (secc. XVII-XVIII), Aracne, Roma.

Dellabarba, S.; Guerreri, E. (2003). Vele italiane della costa occidentale dal medioevo al Novecento, Hoepli, Milano.

Fontenay, M.; Tenenti, A. (2006). "Course et piraterie mèditerranéenses de la fin du moyen âge aux Débuts du XIXe siècle”, Revue d'Histoire Maritime (dedicato a Les Français dans le Pacifique), 6.

Jannucci, G.B.M. (1981). Economia del Commercio nel Regno di Napoli, Assante, F. ed., Giannini, Napoli.

Liberti, R. (2000). "Pirateria e guerra di corsa", Quaderni Mamertini, 17.

Montaudo, A. (2005). L'olio nel Regno di Napoli nel XVIII secolo. Commercio, Annona e Arrendamenti, Edizioni Scientifiche italiane, Napoli.

Mozzillo, A. (1972). Cronache della Calabria in guerra (1806-1811), ESI, Napoli.

Savaglio, A.; Capalbo, M. (2004). Mare Horribilis. Le incursioni musulmane, il mercato degli schiavi e la costruzione delle torri costiere in Calabria Citra, Edizioni Ecofutura, Castrolibero. 